\title{
How do we counsel men with obstructive azoospermia due to CF mutations? - a review of treatment options and outcomes
}

\author{
Jesse B. Persily ${ }^{1}$, Varun Vijay ${ }^{1}$, Bobby B. Najari ${ }^{1,2}$ \\ ${ }^{1}$ Department of Urology, New York University Langone School of Medicine, New York, NY, USA; ${ }^{2}$ Department of Population Health, New York \\ University Langone School of Medicine, New York, NY, USA \\ Contributions: (I) Conception and design: All authors; (II) Administrative support: JB Persily, V Vijay; (III) Provision of study materials or patients: All \\ authors; (IV) Collection and assembly of data: All authors; (V) Data analysis and interpretation: All authors; (VI) Manuscript writing: All authors; (VII) \\ Final approval of manuscript: All authors. \\ Correspondence to: Bobby B. Najari, MD, MSC. NYU Langone Urology Associates, 222 East $41^{\text {st }}$ Street, $11^{\text {th }}$ Floor, New York, NY 10017, USA. \\ Email: Bobby.Najari@nyulangone.org.
}

\begin{abstract}
Obstructive azoospermia $(\mathrm{OA})$ is a rare cause of male infertility, with Congenital Bilateral Absence of The Vas Deferens (CBAVD) being a major cause. A wealth of literature has established an irrefutable link between CFTR mutations and CBAVD, with CBAVD affecting almost all men with cystic fibrosis (CF) disease and a significant portion of men that are CFTR mutation carriers. In the past two decades, assisted reproductive technologies have made the prospect of fathering children a viable possibility in this subset of men, using a combination of sperm extraction techniques and intracystoplasmic sperm injection (ICSI). In order to assess techniques for sperm retrieval, as well as reproductive outcomes, a systemic search of the MEDLINE database was conducted for all articles pertaining to management options for CBAVD, and also all reports describing outcomes of these procedures in the CBAVD population. Both epididymal and testicular sperm extraction (TESE) are viable options for men with CBAVD, and though rigorous data are lacking, live birth rates range from $8 \%$ to $50 \%$ in most small retrospective series and subset analyses. In addition, there does not appear to be significant differences in the rate of live birth or complications and miscarriages between the various techniques, though further investigation into other factors that limit reproductive potential of men with CFTR mutations and CBAVD is warranted.
\end{abstract}

Keywords: Cystic fibrosis (CF); azoospermia; Congenital Bilateral Absence of The Vas Deferens (CBAVD); infertility; testicular sperm extraction (TESE)

Submitted Oct 30, 2019. Accepted for publication May 26, 2020.

doi: 10.21037/tau-19-681

View this article at: http://dx.doi.org/10.21037/tau-19-681

\section{Introduction}

Azoospermia, defined as the absence of sperm in the ejaculate, is identified in up to $15 \%$ of infertile men, and falls into three general categories: pre-testicular azoospermia (often a hormonal issue), testicular (non-obstructive) azoospermia, and post-testicular azoospermia (1). Obstructive azoospermia (OA), a form of post-testicular azoospermia, results from blockage or loss of the male reproductive tract that leads to complete lack of sperm in the ejaculate (2). OA is the cause of azoospermia in up to
$51 \%$ of cases, though epidemiological studies tend to favor non-OA as the slightly more common cause (3-5). Though many causes of non-OA have treatment options, OA lends itself to a broader set of corrective measures (6-9).

OA can be caused by obstruction of the ductal male reproductive tract at any point. Specific causes include both idiopathic and post-vasectomy vasal obstruction, epididymal obstruction, ejaculatory duct obstruction, and absence of the vasa deferentia. OA conditions include Congenital Bilateral Absence of The Vas Deferens (CBAVD), in which segments of the male ductal reproductive tract are absent. 
These missing segments can start anywhere from the midportion of the epididymis (leaving only the head/caput of the epididymis), to the seminal vesicles (10).

CBAVD is present in only roughly $1-2 \%$ of all infertile men, but aside from vasectomy, it is the most common cause of OA (11). The link between CBAVD and mutations in the Cystic Fibrosis Transmembrane-Conductance Regulator (CFTR) gene has been extensively demonstrated (11-13). The CFTR gene is found on chromosome 7 and encodes the CFTR protein, which is the main regulator of an outward flowing chloride channel (14). This protein is critical for salt homeostasis in various epithelial tissues including the lung, pancreas and reproductive tracts (15). Since its discovery in 1989, over 2000 different CFTR mutations have been identified, the most common of which is the F508del mutation, which accounts for approximately two thirds of all abnormal CFTR alleles (16). These mutations have a variety of effects on CFTR function, which have resulted in an evergrowing number of broad classes of mutations, and an even wider range of patient phenotypes $(12,17)$. Adding to the diversity, the disease presents differently in the heterozygous and homozygous forms. When a mutant allele of the CFTR gene is passed down from both parents, the child can develop cystic fibrosis (CF), a debilitating multi-organ disease involving the lungs, pancreas, gastrointestinal, and reproductive system. Compound heterozygous individuals, who receive two different mutant alleles, typically present with an intermediate or variable phenotype (18). When only one allele containing a CFTR mutation is passed down from one parent, the child will be a CF carrier and may have mild or completely absent symptoms (12).

Nearly all (roughly 98\%) of patients with symptomatic CF are noted to have CBAVD on physical exam $(19,20)$. Though there are cases of CBAVD that are not associated with CFTR mutations, $80-97 \%$ of patients with CBAVD have identifiable mutations in the CFTR gene. Additionally, CBAVD is associated with hypoplastic seminal vesicles, seminal hypovolemia and acidic ejaculate $(\mathrm{pH}<7)(20)$. For these reasons, these patients historically had poor fertility outcomes.

With the marked increase in life expectancy in patients with CF disease and advances in assistive reproductive technology (ART) over the past 2 decades, there has been increased interest in fertility treatment in men with OA due to CF mutations and men diagnosed with CBAVD. Men in these categories who seek biological fertility must undergo surgical collection of sperm, as very few of these men have enough healthy/present tissue to make reconstruction of the tract possible (21). Over the past two decades, the surgical options of sperm extraction have expanded and been refined to optimize reproductive outcomes (22). After successful sperm extraction, sperm can be used for fertilization of the oocyte using in vitro fertilization (IVF) and intracytoplasmic sperm injection (ICSI), or frozen for later use.

Despite the relatively rapid rise in sperm retrieval options, there is still a paucity of rigorous outcomes data evaluating the available surgical options for men with OA due to CFTR gene mutations. This review will distill down the diagnostic considerations, treatment options, and existing outcomes data for OA in men with CBAVD, empowering physicians to better counsel men with CBAVD about why their experience with sperm retrieval and pregnancy may differ from other men with OA.

\section{Diagnostic considerations: OA, CF, and CBAVD}

Infertile men are at risk for a wide range of genetic abnormalities, including but not limited to mutations in the CFTR gene, and as such, specific genetic tests should be based on the clinical context. Relevant to the current discussion, initial assessment of infertile men must attempt to differentiate between obstructive and nonobstructive causes using a combination of history, physical exam including a thorough scrotal and vasal exam, semen analysis and serum lab tests in order to look for potentially correctable hormonal abnormalities (23).

OA patients have normal testis volume and normal levels of FSH, indicating a functional hypothalamic-pituitarygonadal axis. Men with CBAVD will have reduced ejaculate volume, typically less than $1 \mathrm{~mL}$, due to their hypoplastic seminal vesicles (24). While men with OA have normal spermatogenesis, testicular biopsy is not necessary to make the empiric diagnosis of OA $(25,26)$. Diagnostic biopsy should instead be considered when there is clinical uncertainty whether the patient has obstructive or non-OA or when the patient has risk factors for testicular germ cell tumors (family history, history of cryptorchidism, suggestive US characteristics) $(27,28)$ In the setting of OA with low semen volume, CFTR genetic analysis is always warranted, even if the vasa are present, because reproductive tract abnormalities secondary to CFTR mutations are not limited to the vasa (29).

Upon physical exam, CBAVD patients may not only have absent vasa deferentia, but may also have absent epididymal segments or irregular or indurated epididymal tissue. However, the caput of the epididymis is of different 
embryologic origin and is almost always present in men with CBAVD (30). In men with Congenital Unilateral Absence of the Vas Deferens (CUAVD), renal ultrasound is performed to rule out renal agenesis, because concomitant vasal abnormalities and renal agenesis is indicative of a broader mesonephric duct abnormality that is not secondary to CFTR mutations (31-33). Renal ultrasound should also be performed in men with CBAVD without identified CFTR mutations (28).

While a complete discussion of the CFTR mutation profile of CBAVD patients is beyond the scope of this review and is discussed in more depth in another article in this focused issue, a few key points are worth noting. As was stated earlier, men with clinical CF nearly always present with CBAVD, while anywhere from $78-97 \%$ of men with CBAVD are found to have at least one CFTR mutation (12). However, patients may present with two CFTR mutations but not have clinical features of CF aside from CBAVD. It is hypothesized that these phenotypic difference are related to alternative mRNA splicing in different tissues, and specifically inefficient splicing in the vas deferens that locally compromises CFTR function $(34,35)$. When CBAVD is the only presenting feature in a patient with at least one CFTR mutations, the disease is sometimes referred to as the joint CF-CBAVD. These presentations are often characterized by higher frequencies of atypical CFTR gene variants, including the IVS8-5T polymorphism, the TG variant, and more frequent class IV and V CFTR mutations.

\section{Treatment options}

Many forms of OA, especially OA secondary to vasectomy, are amenable to microsurgical tract reconstruction. The technical aspects of these procedures are the focus of other reviews $(36,37)$. However, cases of reconstructive failure and OA not amenable to reconstruction were often considered hopeless until the advent and widespread adoption of ART in the early 1990s. A number of procedures have been developed and refined that allow for extraction of sperm from the epididymis and testicles. These techniques include microscopic epididymal sperm aspiration (MESA), percutaneous epididymal sperm aspiration (PESA), testicular sperm extraction (TESE), and percutaneous testicular sperm aspiration (TESA). Of note, sperm obtained from chronically obstructed duct systems, or prior to complete maturation in the epididymis, tend to have poor motility, and thus, retrieved sperm from CBAVD patients should be used for ICSI, and not intra-uterine injection or other IVF methods.

\section{MESA}

Epididymal sperm offers the advantage of longer transit and maturation time relative to testicular sperm. MESA in particular offers the advantage of sperm aspiration under direct vision of the epididymal tubules (38). Through a small scrotal incision, the testis is delivered, the tunica vaginalis is opened, and the epididymis is inspected under 16-25x magnifications using the operating microscope. The standard approach is to search for superficial dilated tubules, make small incisions to extract fluid, and check the fluid for sperm under a microscope. If no sperm are found, the process is repeated at a more proximal part of the epididymis (39). Of course, in cases of CBAVD when only the caput of the epididymis is present, the relatively limited real estate for extraction must be taken into account. Gentle compression of the testis and epididymis can enhance flow from the incised tubule. Sperm can then be cryopreserved in multiple aliquots so that several ICSI cycles can be attempted.

\section{PESA}

Though PESA is considered by some to be less reliable than open epididymal sperm retrieval, given that the small quantities of sperm are sometimes inadequate for cryopreservation, reported pregnancy rates in general are similar to those achieved with open techniques without requiring microsurgical skill (40). A recent surgical video highlighted the simplicity of this technique, which involves the use of a hypodermic needle attached to a $1 \mathrm{cc}$ syringe, which is inserted through the skin into the corpus or caput of the epididymis and used to aspirate and then quickly analyze sperm content (41). The blind approach can necessitate multiple passes, increasing the risk for scrotal hematoma. Given the increased risk, and potential for lower quantity of sperm, epididymal sperm retrieval under direct vision is considered the preferred technique by many, especially in the context of the cost and effort that goes into IVF to begin with $(42,43)$. That said, MESA entails the cost associated with the use of the surgical microscope as well as longer time under anesthesia, and thus, both procedures have pros and cons. 


\section{TESA and TESE}

TESA or TESE are typically indicated when there is a failure to find sperm in the epididymis, or in some cases of CBAVD, when there is not enough viable epididymis present. TESA is the least invasive method, though it may require 10-20 passes using a high-suction glass syringe and a 23 -gauge needle $(44,45)$. TESA has the same technical advantages as PESA, in that no microsurgical skill is required, and only local anesthesia is used. The drawback of these techniques is that testicular sperm tends to be immature and immobile because of the lack of epididymal maturation. As with other blind procedures, the risk of hematoma, vascular injury, and post-procedure pain are increased with TESA.

TESE, and testicular biopsy in general, can be helpful for distinguishing OA from non-OA, though if OA is suspected, testicular biopsy is not required before sperm retrieval and ICSI are attempted (46). TESE, and microsurgical TESE (micro-TESE), offer the same advantage as MESA, in that extraction can be performed under direct vision. Open TESE, and micro-TESE in particular, allows retrieval of a large number of sperm for potential cryopreservation and the use for multiple cycles of ICSI.

The diversity of options has drastically changed both the management of and fertility-related outcomes in men with CBAVD. Given the phenotypic range in this population, and in particular, the range in length of viable epididymal tissue, TESE in combination with ICSI have maximized the options available those who provide infertility services to these couples. However, given the superior maturity of epididymal sperm, a comparison of outcomes, both in terms of sperm retrieval rates and also pregnancy-related outcomes, is warranted.

\section{Sperm retrieval and pregnancy-related outcomes in the CF/CBAVD population}

Unfortunately, very few studies were designed to look specifically at sperm retrieval outcomes in the CF/CBAVD population. Many of the studies to be presented do fortunately provide subset analyses that look specifically at men in the study with these conditions. However, very few speak specifically about the variation in CF mutations present, and many of the sample sizes were made quite small by the subset analyses. There are no randomized controlled trials that attempt to answer which method for sperm retrieval is superior, and the studies consist of mainly retrospective analysis, case-control studies, and a few prospective studies. Additionally, because the goal is always adequate sperm retrieval and subsequent fertilization, implantation, and a successful pregnancy, many studies consider not only a primary technique of retrieval, but also a reflex secondary technique. These studies will be discussed under both relevant sub-sections. Sperm retrieval rates and outcomes are presented in Table 1. ICSI/pregnancy outcomes are presented in Table 2 .

\section{MESA}

A group led by Schroeder-Printzen et al. was one of the earliest to publish robust sperm retrieval data for men with non-reconstructable $\mathrm{OA}$, a group that likely included men with CBAVD, though the paper did not specify (47). Of the 93 men in the cohort that underwent MESA, sperm were retrieved from 88 of them, a retrieval rate of $94.6 \%$. Mean sperm concentration was $40.9 \times 10^{6} / \mathrm{mL}$, and global motility rate was on average $24.8 \%$. This group also achieved a pregnancy rate of $42.4 \%$ after 33 ICSI cycles with frozen sperm.

Silber and colleagues were the first to publish more specifically on the use of MESA for CBAVD, and also on the outcomes of ICSI in men with CBAVD $(55,59,60)$. Given that these reports were made during the early days of ICSI, the initial reports focused on a comparison between MESA followed by IVF and MESA followed by IVF/ICSI, but the IVF/ICSI data will be highlighted. The group first published a series of 72 consecutive MESA followed by IVF/ ICSI cases $(55,59)$. ICSI resulted in fertilization and embryo transfer in $90 \%$ of cases, leading to an ongoing or delivered pregnancy rate of $46 \%$ per transfer and $42 \%$ per cycle. Notably, the pregnancy and live-birth baby rates were 53\% and $42 \%$ respectively with ICSI. Similarly, Hubert et al. looked at 23 men with CF who chose to proceed with sperm retrieval and IVF/ICSI and found a similar pregnancy rate per cycle $(40 \%)$, clinical pregnancy rate $(63 \%)$ and livebirth rate $(47 \%)$, concluding that this technique can be an effective option in men with CBAVD (48).

McCallum and colleagues conducted a retrospective analysis looking at MESA outcomes specifically in men with the F508del CFTR mutation (50). Of the nine men, eight underwent MESA, five couples went on to achieve a pregnancy, and four couples delivered seven kids. This equated to a fertilization rate of $75 \%$ and a life birth rate per cycle of $43.75 \%$. Interestingly, the author's key take away was that physicians involved in the reproductive care 
Table 1 Sperm retrieval outcomes

\begin{tabular}{|c|c|c|c|c|c|c|}
\hline Study & $\begin{array}{l}\text { Number of } \\
\text { procedures }\end{array}$ & CBAVD only? & $\begin{array}{c}\text { Sperm Retrieval } \\
\text { rate, } \%\end{array}$ & $\begin{array}{l}\text { Amount of sperm } \\
\quad(\text { per } \mathrm{mL})\end{array}$ & $\%$ Motile & $\%$ Cryo $^{a}$ \\
\hline \multicolumn{7}{|l|}{ MESA } \\
\hline Hubert 2006 (48) & 23 & YES & 82.60 & $\mathrm{~N} / \mathrm{A}$ & N/A & N/A \\
\hline Yamamoto 1997 & 32 & YES & 100 & $25.1 \times 10^{6}$ & 34.50 & $\mathrm{~N} / \mathrm{A}$ \\
\hline Llabador 2015 (51) & 47 & YES & 43.5 & N/A & N/A & $\mathrm{N} / \mathrm{A}$ \\
\hline Janzen 2000 (42) & 141 & NO & 100.0 & $\mathrm{~N} / \mathrm{A}$ & 100 & 23.40 \\
\hline Esteves 2015 (41) & 32 & YES & 98.60 & N/A & N/A & 21.90 \\
\hline Semião-Francisco 2010 (54) & 171 & NO & 100 & N/A & 93.60 & N/A \\
\hline \multicolumn{7}{|l|}{ TESE } \\
\hline Llabador 2015 (51) & 61 & YES & 100 & N/A & N/A & N/A \\
\hline \multicolumn{7}{|l|}{ TESA } \\
\hline Semião-Francisco 2010 (54) & 103 & NO & 100 & N/A & 58.90 & $\mathrm{~N} / \mathrm{A}$ \\
\hline Glina et al. 2003 (52) & 14 & NO & 100 & N/A & N/A & N/A \\
\hline
\end{tabular}

a $\%$ of men with retrieved sperm who proceeded with cryopreservation. ICSI, intracystoplasmic sperm injection; CBAVD, congenital bilateral absence of the vas deferens; MESA, microscopic epididymal sperm aspiration; PESA, percutaneous epididymal sperm aspiration; TESE, testicular sperm extraction; TESA, testicular sperm aspiration.

of CF patients should be optimistic about the prospects of fatherhood, which they considered "excellent" given the current state of the technology even in 2000.

Finally, Llabador and colleagues looked at both MESA and TESE used to retrieve sperm from men with CBAVD (51). The sperm retrieval rate for MESA alone was $43.5 \%$. They did find an overall fertilization rate of $67.1 \%$, an implantation rate of $15.3 \%$, and clinical pregnancy and birth rates of $26.5 \%$ and $22 \%$, respectively. It is interesting to note that these authors found differences in spermatogenesis quality within this cohort of men with CBAVD, and that poor quality predicted poor fertility outcomes. This variation may also contribute to the low sperm retrieval rates, but it is unclear why this cohort differed from the cohorts in other studies.

Given these results, MESA appears to be a viable option for men with CBAVD seeking infertility treatment and sperm extraction, despite concern about variable quality epididymal tissue. Though sperm retrieval outcomes were insufficiently reported in many cases, most groups were able to retrieve sperm in roughly $80-100 \%$ of men. IVF/ICSI is a viable option for couples where the man has CBAVD, with fertilization rates between $60 \%$ and $75 \%$, and live birth rates per cycle between $18 \%$ and $45 \%$. Standard reporting of fertility and pregnancy outcomes would have aided the analysis, as would prospective study designs.

\section{PESA}

Though MESA is still considered the gold standard for epididymal sperm retrieval, a number of studies have assessed the viability of PESA as a less invasive alternative for sperm retrieval in CBAVD patients. Lu and colleagues completed a single center retrospective analysis of 945 
Table 2 ICSI/pregnancy outcomes

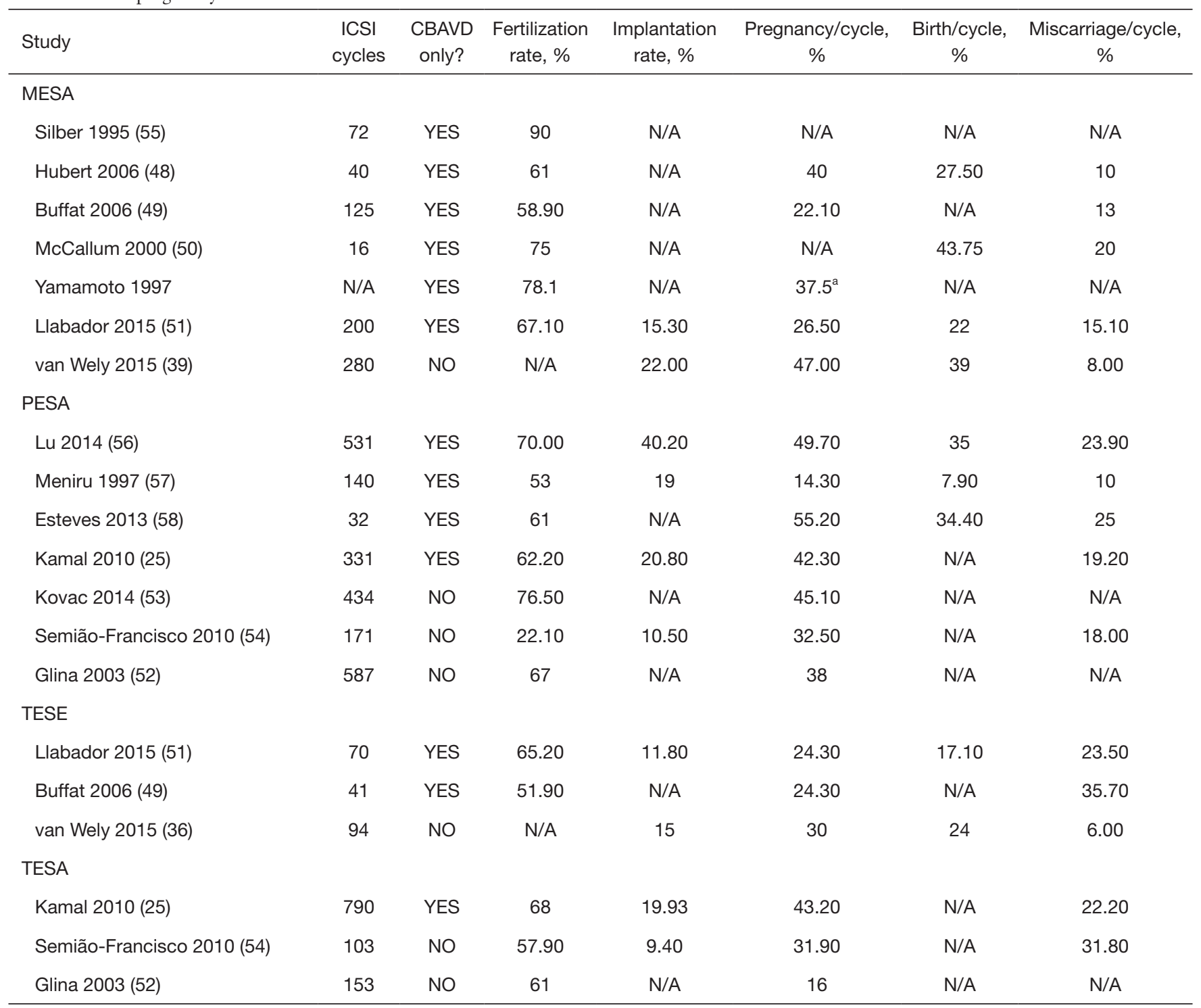

a, pregnancy rate per couple was the only statistic reported. ICSI, intracystoplasmic sperm injection; CBAVD, congenital bilateral absence of the vas deferens; MESA, microscopic epididymal sperm aspiration; PESA, percutaneous epididymal sperm aspiration; TESE, testicular sperm extraction; TESA, testicular sperm aspiration.

patients and 1,414 ICSI cycles, with the goal of comparing ICSI outcomes in men with CBAVD and men with acquired OA. The group found significantly lower rates of live birth per embryo transferred, and significantly higher rates of miscarriage and ectopic pregnancy per embryo transferred in the CBAVD group (56). The authors hypothesized that the higher rates of CFTR mutations in the CBAVD group may have explained the increased rates of miscarriage and stillbirth. In this group of Chinese men, only $13 \%$ of men with CBAVD had CFTR mutations compared to Western studies which demonstrate approximately $78 \%$ to $97 \%$ of CBAVD patients harbor at least one CFTR mutation $(19,61)$. The authors suggest that the prevalence and/or type of CFTR mutation may have a clinical impact on fertility outcomes.

In an older, smaller retrospective review, Meniru and colleagues compared men with CBAVD to men with failed vasectomy reversal using PESA and that the pregnancy rates between the groups did not significantly differ (14.3\% for the CBAVD group vs. $12.1 \%$ for the non-CBAVD 
group) sperm (57). Similarly, Esteves et al. retrospectively compared PESA outcomes based on the cause of OA in 146 men in a single center, and found a $96.8 \%$ sperm retrieval rate with PESA alone in CBAVD patients, while only $3.2 \%$ of men with CBAVD required rescue TESA after PESA (58). The group achieved clinical pregnancy in 16 men (55.2\%) of the CBAVD group, resulting in 11 live births, one still birth, and 4 miscarriages.

An additional study by Kamal and colleagues sought to assess whether the cause of OA affected ICSI outcomes, using both PESA and TESA depending on the situation. TESA outcomes will be discussed later. PESA resulted in 331 ICSI cycles in men with CBAVD, resulting in a fertilization rate of $62.2 \%$, an implantation rate of $20.8 \%$, a rate of clinical pregnancy of $42.3 \%$, yet a rate of miscarriage of $19.2 \%$. These numbers were similar to a study by Glina and colleagues, which examined the results of 587 ICSI cycles and found a $67 \%$ fertilization rate and a $38 \%$ clinical pregnancy rate per implantation cycle. However, this study was not limited to CBAVD causes of OA (52).

Thus, though MESA is still considered by many to be the gold standard for sperm retrieval, PESA is an additional viable option for sperm retrieval. The studies did not speak to complication rate, and rarely mentioned the challenge of completing PESA in men with less substantial epididymal tissue, and so again, standardizing outcomes and discussing sperm retrieval characteristics relative to the specific nature of the CBAVD anatomy would aid in the development of a more robust comparative framework for the two procedures. In addition, only one group attempted to compare outcomes of fresh vs. frozen sperm, another possible useful line of inquiry that has yet to be adequately studied in the literature (53). The last challenge in making a definitive statement is that many of the comparative studies on this subject focus on epididymal $v s$. testicular sperm, but few if any focus on different retrieval methods for sperm from the same location. Given the aforementioned differences in procedure morbidity and invasiveness, these procedures warrant further comparison. In terms of available data, retrieval rates with PESA do not seem substantially inferior to those with MESA, though there appears to be greater variability in fertilization rates with PESA. Though the studies are weak, clinical pregnancy rates and life birth rates also do not appear to differ between PESA and MESA.

\section{TESE}

Few studies have reported CBAVD-specific TESE outcomes, though it is important to note that there is data going back as far as 1995 that testicular sperm can be as effective as epididymal sperm when combined with ICSI (55). Llabador et al. looked at MESA and TESE, because in $43.5 \%$ of cases, MESA was followed by TESE in the same surgical session because no sperm were found in the epididymis (51). Sperm was retrieved in all 61 men who had reflex TESE performed. The group found a non-significant difference in the delivery rate $(27 \%$ with MESA and $18.6 \%$ with TESE), and also a non-significant difference in miscarriage rate $(15.1 \%$ with MESA and $23.5 \%$ with TESE), though there was a trend pointing to the superiority of MESA. Of note, most clinical pregnancies required no more than two ICSI cycles, despite the relative immaturity of testicular sperm.

Buffat et al. compared outcomes using epididymal and testicular extraction methods, and found that fertilization and clinical pregnancy rates did not differ between the two. The group found that the miscarriage rate was statistically significantly higher in the TESE group than in the MESA group $37.5 \%$ in the TESE group vs. the $12.5 \%$ in the MESA group). Given the increased miscarriage rate, the group concluded that testicular sperm lacked some characteristic required to ensure a viable pregnancy, and that epididymal sperm should be used when available (49).

Though lacking CBAVD stratification, a useful comparison between MESA and TESE was conducted by van Wely et al. (39). The analysis included 374 total ICSI cycles and found MESA had a superior live birth rate (39\% from MESA vs. 24\% for TESE), and that MESA had an adjusted multivariable logistic analysis OR of 1.82 (95\% CI: 1.05-3.67) for on-going pregnancy compared to microTESE. Despite this large sample size, and a clear distinction in the text about CBAVD patients, no sub-analysis was carried out, making a direct assessment of CBAVD/CF outcomes difficult.

The paucity of data in men with OA and then specifically CBAVD-OA makes drawing conclusions about the use of TESE for CBAVD patients difficult. Some data suggests slightly lower fertilization rates, lower clinical pregnancy rates, and higher rates of miscarriage with testicular sperm compared to epididymal sperm. However, these results are based on limited comparative data and more rigorously designed comparative studies are warranted and necessary.

\section{TESA}

CBAVD specific TESA outcomes are rarely reported in 
the literature. Kamal et al. performed TESA followed by 221 cycles of ICSI using sperm from men with CBAVD, and found a fertilization rate of $68.2 \%$, an implantation rate of $21.56 \%$, a clinical pregnancy rate of $46.2 \%$, but a miscarriage rate of $22.2 \%$. Semião-Francisco and colleagues conducted a retrospective analysis that did not stratify based on CBAVD, but included 103 total ICSI cycles and found a sperm retrieval rate of $100 \%$ (54). Unfortunately, pregnancy outcomes were not stratified by CBAVD. However, in the same study, the group compared TESA to PESA, and found that, though the fertilization rate was greater for PESA, when retrieved sperm were used for ICSI, there was no difference in clinical pregnancy and implantation rates. Glina and colleagues also conducted a retrospective analysis of men with OA, however they reported that the most common cause was vasectomy, and did not specify the causes in other cases. Motile sperm were only found in 65 of 79 PESA procedures, and the remaining 14 men had to undergo salvage TESA (52). Thus, CF/CBAVD outcomes specific to TESA are rare, but the literature may suggest, based on miscarriage rate alone, that epididymal sperm is superior when available in patients with OA.

As a final point about all four procedures, though sperm retrieval outcomes are presented in Table 1, retrieval rates and outcomes in this population are severely lacking in the literature. Even papers about the efficacy and feasibility of the procedure failed to report standard sperm parameters, including amount of sperm and motility, in many cases. Additionally, though there are papers comparing fresh and frozen sperm from these various procedures in other populations, and also CBAVD, few papers reported the rates at which couples decided to cryopreserve sperm, or the rates at which sufficient tissue was obtained for cryopreservation, severely limiting the interpretation and comparison of sperm retrieval rates between the different techniques in the CBAVD population (53).

\section{Discussion and future directions}

Despite rapid and robust developments in the genetic characterization of CBAVD, there is a dearth of specific fertility outcomes on this sub-set of men. From the limited data, it appears as though the literature demonstrates that all sperm retrieval modalities are reasonable options for men presenting with CBAVD, given that groups have had success with all techniques. Men should be counseled that testicular sperm may have slightly lower rates of pregnancy compared to epididymal sperm, keeping in mind that the amount of viable epididymal tissue differs within this cohort of men.

To illustrate the gaps in the literature, a number of additional studies warrant discussion despite their lack of a CBAVD focus. A study by Naru et al. compared PESA and TESE, but failed to mention CBAVD despite the fact that a proportion of men in any cohort of OA would be expected to have CBAVD (62). Bernie and colleagues presented the pros and cons of MESA in the context of other OA and non-OA treatment modalities. The article included a pertinent review of technique modification, such as changing the gauge of the needle used for TESA (small needle, large needle, and core needle), and provided a more granular review of TESE vs. micro-TESE (45,63-65). Though this paper's useful summary presented sperm retrieval rates between 95 and $100 \%$, as well as sperm yields for each technique, it did not stratify by origin of OA or between non-OA and OA. In addition, a 2008 meta-analysis on the topic, which did not find a significant difference in efficacy between testicular and epididymal retrieval techniques, did not stratify by origin of $\mathrm{OA}$, and noted above all the lack of high quality comparative, randomized, and controlled data on the topic (66). Thus, given the general clinical equipoise up to this point, future studies in this cohort should seek to prospectively enroll men with CBAVD to not only directly compare testicular and epididymal techniques, but also compare the different methods of extraction at each site. The studies must publish robust reproductive outcomes, including quantity of sperm retrieved, number of ICSI cycles, and granular pregnancy outcomes, so that providers have access to a more nuanced set of outcomes data when counseling their patients.

In addition, an early meta-analysis raised the important question as to why, independent of method of sperm retrieval, CBAVD patient may have reduced fertility relative to other patients with OA. The leading theory is that a subset of CBAVD patients may have a degree of diminished spermatogenesis, resulting in decreased sperm quality, regardless of sperm retrieval modality $(67,68)$. Previous literature has elucidated the role of the CFTR gene in various aspects of spermatogenesis. Men with CBAVD who underwent ICSI were more likely to have miscarriage or still birth and less likely to have a live birth, indicating that $C F T R$ mutation is potentially associated with sperm function (56). Higher rates of CFTR mutations have been found in men with poor semen quality $(56,67,69,70)$. At least 5 different CFTR mutations have been linked with non- OA or oligozoospermia (68). While the underlying mechanism is not fully understood, the CFTR gene is thought to be a key regulator in maintaining the luminal microenvironment of 
the male reproductive tract, which in turn has an impact on spermatogenesis and sperm maturation $(68,71)$.

A related issue, brought into focus by the conflicting results from the study by $\mathrm{Lu}$ and colleagues, is that many of these studies were done at a time where the panel of CFTR mutations that was regularly tested was not nearly as expansive as it is now. This issue, as well as regional and ethnic differences in mutational frequency and incomplete characterization of CBAVD as a systematic disease entity, limits the conclusions that can be drawn about mutational variants and their effect on reproductive outcomes. In addition, discussions of fertility management in patients with CFTR mutations will continue to make their way into the greater reproductive ethics and ethics of assisted reproductive technologies discussion, as people wrestle with the fact that individuals with an increasingly wide range of phenotypic presentations of CF, who were once considered infertile, can now be rendered fertile through the help of these ever expanding technologies. This discussion will invariably include whether or not the female partners of these men should under CFTR mutation analysis. This may be even more important in men with CBAVD and no identified common CFTR mutations, given that they may have CFTR mutations that are less commonly tested for.

\section{Conclusions}

As assisted reproductive technologies continue to develop for both men and women, the reproductive prospect for patient with CF/CBAVD has shifted dramatically, from that of a state of complete infertility, to the current state of the field, where OA can be managed with a spectrum of interventions. As these techniques continue to be employed, additional rigorous retrospective analyses, as well as prospective comparative trials, will be necessary to determine whether there is an ultimately superior technique that should be offered to men with CBAVD. Though current data point toward the use of epididymal sperm, testicular sperm still provides a viable option for reproduction, and the possibility of pregnancy loss should be discussed, regardless of the source of sperm. The future continues to look bright for these men, though there is still much to be learned about the ever-increasing number of CFTR mutations and their impact on male fertility.

\section{Acknowledgments}

Funding: None.

\section{Footnote}

Provenance and Peer Review: This article was commissioned by the Guest Editors (Keith Jarvi and Jared Bieniek) for the series "Genetic Causes and Management of Male Infertility" published in Translational Andrology and Urology. The article was sent for external peer review organized by the Guest Editors and the editorial office.

Conflicts of Interest: All authors have completed the ICMJE uniform disclosure form (available at http://dx.doi. org/10.21037/tau-19-681). The series "Genetic Causes and Management of Male Infertility" was commissioned by the editorial office without any funding or sponsorship. The authors have no other conflicts of interest to declare.

Ethical statement: The authors are accountable for all aspects of the work in ensuring that questions related to the accuracy or integrity of any part of the work are appropriately investigated and resolved.

Open Access Statement: This is an Open Access article distributed in accordance with the Creative Commons Attribution-NonCommercial-NoDerivs 4.0 International License (CC BY-NC-ND 4.0), which permits the noncommercial replication and distribution of the article with the strict proviso that no changes or edits are made and the original work is properly cited (including links to both the formal publication through the relevant DOI and the license). See: https://creativecommons.org/licenses/by-nc-nd/4.0/.

\section{References}

1. Cocuzza M, Alvarenga C, Pagani R. The epidemiology and etiology of azoospermia. Clinics (Sao Paulo) 2013;68 Suppl 1:15-26.

2. Jarvi K, Lo K, Grober E, et al. The workup and management of azoospermic males. Can Urol Assoc J 2015;9:229-35.

3. Fogle RH, Steiner AZ, Marshall FE, et al. Etiology of azoospermia in a large nonreferral inner-city population. Fertil Steril 2006;86:197-9.

4. Fedder J, Cruger D, Oestergaard B, et al. Etiology of azoospermia in 100 consecutive nonvasectomized men. Fertil Steril 2004;82:1463-5.

5. Jarow JP, Espeland MA, Lipshultz LI. Evaluation of the azoospermic patient. J Urol 1989;142:62-5.

6. Colpi GM, Piediferro G, Nerva F, et al. Sperm retrieval 
for intra-cytoplasmic sperm injection in non-obstructive azoospermia. Minerva Urol Nefrol 2005;57:99-107.

7. Cocuzza M, Athayde KS, Agarwal A, et al. Impact of clinical varicocele and testis size on seminal reactive oxygen species levels in a fertile population: a prospective controlled study. Fertil Steril 2008;90:1103-8.

8. Watkin NA, Reiger NA, Moisey CU. Is the conservative management of the acute scrotum justified on clinical grounds? Br J Urol 1996;78:623-7.

9. Visser AJ, Heyns CF. Testicular function after torsion of the spermatic cord. BJU Int 2003;92:200-3.

10. Daudin M, Bieth E, Bujan L, et al. Congenital bilateral absence of the vas deferens: clinical characteristics, biological parameters, cystic fibrosis transmembrane conductance regulator gene mutations, and implications for genetic counseling. Fertil Steril 2000;74:1164-74.

11. Jequier AM, Ansell ID, Bullimore NJ. Congenital absence of the vasa deferentia presenting with infertility. J Androl 1985;6:15-9.

12. de Souza DAS, Faucz FR, Pereira-Ferrari L, et al. Congenital bilateral absence of the vas deferens as an atypical form of cystic fibrosis: reproductive implications and genetic counseling. Andrology 2018;6:127-35.

13. Hussein TM, Zakaria NH, Zahran AM. Clinical, laboratory and genetic assessment of patients with congenital bilateral absent vas deferens. Andrologia 2011;43:16-22.

14. Anguiano A, Oates RD, Amos JA, et al. Congenital bilateral absence of the vas deferens. A primarily genital form of cystic fibrosis. Jama 1992;267:1794-7.

15. Reisin IL, Prat AG, Abraham EH, et al. The cystic fibrosis transmembrane conductance regulator is a dual ATP and chloride channel. J Biol Chem 1994;269:20584-91.

16. Castellani C, Cuppens H, Macek M, Jr., et al. Consensus on the use and interpretation of cystic fibrosis mutation analysis in clinical practice. J Cyst Fibros 2008;7:179-96.

17. Veit G, Avramescu RG, Chiang AN, et al. From CFTR biology toward combinatorial pharmacotherapy: expanded classification of cystic fibrosis mutations. Mol Biol Cell 2016;27:424-33.

18. Xie Y, Huang X, Liang Y, et al. A new compound heterozygous CFTR mutation in a Chinese family with cystic fibrosis. Clin Respir J 2017;11:696-702.

19. Chillón M, Casals T, Mercier B, et al. Mutations in the cystic fibrosis gene in patients with congenital absence of the vas deferens. N Engl J Med 1995;332:1475-80.

20. Attardo T, Vicari E, Mollica F, et al. Genetic, andrological and clinical characteristics of patients with congenital bilateral absence of the vas deferens. Int J Androl 2001;24:73-9.

21. Stuhrmann M, Dork T. CFTR gene mutations and male infertility. Andrologia 2000;32:71-83.

22. Coward RM, Mills JN. A step-by-step guide to officebased sperm retrieval for obstructive azoospermia. Transl Androl Urol 2017;6:730-44.

23. Bhasin S, de Kretser DM, Baker HW. Clinical review 64: Pathophysiology and natural history of male infertility. J Clin Endocrinol Metab 1994;79:1525-9.

24. Boucher D, Creveaux I, Grizard G, et al. Screening for cystic fibrosis transmembrane conductance regulator gene mutations in men included in an intracytoplasmic sperm injection programme. Mol Hum Reprod 1999;5:587-93.

25. Kamal A, Fahmy I, Mansour R, et al. Does the outcome of ICSI in cases of obstructive azoospermia depend on the origin of the retrieved spermatozoa or the cause of obstruction? A comparative analysis. Fertil Steril 2010;94:2135-40.

26. Lee R, Goldstein M, Ullery BW, et al. Value of serum antisperm antibodies in diagnosing obstructive azoospermia. J Urol 2009;181:264-9.

27. van Casteren NJ, Looijenga LH, Dohle GR. Testicular microlithiasis and carcinoma in situ overview and proposed clinical guideline. Int J Androl 2009;32:279-87.

28. Practice Committee of the American Society for Reproductive Medicine in collaboration with the Society for Male Reproduction and Urology. Evaluation of the azoospermic male: a committee opinion. Fertil Steril. 2018;109:777-82.

29. Wosnitzer MS. Genetic evaluation of male infertility. Transl Androl Urol 2014;3:17-26.

30. Joseph A, Yao H, Hinton BT. Development and morphogenesis of the Wolffian/epididymal duct, more twists and turns. Dev Biol 2009;325:6-14.

31. Oates RD, Amos JA. The genetic basis of congenital bilateral absence of the vas deferens and cystic fibrosis. $J$ Androl 1994;15:1-8.

32. McCallum T, Milunsky J, Munarriz R, et al. Unilateral renal agenesis associated with congenital bilateral absence of the vas deferens: phenotypic findings and genetic considerations. Hum Reprod 2001;16:282-8.

33. McPherson E, Carey J, Kramer A, et al. Dominantly inherited renal adysplasia. Am J Med Genet 1987;26:863-72.

34. Cuppens H, Cassiman JJ. CFTR mutations and polymorphisms in male infertility. Int J Androl 2004;27:251-6.

35. Mak V, Jarvi KA, Zielenski J, et al. Higher proportion of 
intact exon 9 CFTR mRNA in nasal epithelium compared with vas deferens. Hum Mol Genet 1997;6:2099-107.

36. Miyaoka R, Esteves SC. Predictive factors for sperm retrieval and sperm injection outcomes in obstructive azoospermia: do etiology, retrieval techniques and gamete source play a role? Clinics (Sao Paulo) 2013;68 Suppl 1:111-9.

37. Practice Committee of the American Society for Reproductive Medicine in collaboration with the Society for Male Reproduction and Urology. Electronic address: asrm@asrm.org. The management of obstructive azoospermia: a committee opinion. Fertil Steril 2019;111:873-80.

38. Matthews GJ, Goldstein M. A simplified method of epididymal sperm aspiration. Urology 1996;47:123-5.

39. van Wely M, Barbey N, Meissner A, et al. Live birth rates after MESA or TESE in men with obstructive azoospermia: is there a difference? Hum Reprod 2015;30:761-6.

40. Sheynkin YR, Ye Z, Menendez S, et al. Controlled comparison of percutaneous and microsurgical sperm retrieval in men with obstructive azoospermia. Hum Reprod 1998;13:3086-9.

41. Esteves SC. Percutaneous epididymal sperm aspiration as a method for sperm retrieval in men with obstructive azoospermia seeking fertility: operative and laboratory aspects. Int Braz J Urol 2015;41:817; discussion 818.

42. Janzen N, Goldstein M, Schlegel PN, et al. Use of electively cryopreserved microsurgically aspirated epididymal sperm with IVF and intracytoplasmic sperm injection for obstructive azoospermia. Fertil Steril 2000;74:696-701.

43. Anger JT, Wang GJ, Boorjian SA, et al. Sperm cryopreservation and in vitro fertilization/ intracytoplasmic sperm injection in men with congenital bilateral absence of the vas deferens: a success story. Fertil Steril 2004;82:1452-4.

44. Rajfer J, Binder S. Use of biopty gun for transcutaneous testicular biopsies. J Urol 1989;142:1021-2.

45. Craft I, Tsirigotis M, Bennett V, et al. Percutaneous epididymal sperm aspiration and intracytoplasmic sperm injection in the management of infertility due to obstructive azoospermia. Fertil Steril 1995;63:1038-42.

46. Goldstein M, Schlossberg S. Men with congenital absence of the vas deferens often have seminal vesicles. J Urol 1988;140:85-6.

47. Schroeder-Printzen I, Zumbe J, Bispink L, et al. Microsurgical epididymal sperm aspiration: aspirate analysis and straws available after cryopreservation in patients with non-reconstructable obstructive azoospermia. MESA/TESE Group Giessen. Hum Reprod 2000;15:2531-5.

48. Hubert D, Patrat C, Guibert J, et al. Results of assisted reproductive technique in men with cystic fibrosis. Hum Reprod 2006;21:1232-6.

49. Buffat C, Patrat C, Merlet F, et al. ICSI outcomes in obstructive azoospermia: influence of the origin of surgically retrieved spermatozoa and the cause of obstruction. Hum Reprod 2006;21:1018-24.

50. McCallum TJ, Milunsky JM, Cunningham DL, et al. Fertility in men with cystic fibrosis: an update on current surgical practices and outcomes. Chest 2000;118:1059-62.

51. Llabador MA, Pagin A, Lefebvre-Maunoury C, et al. Congenital bilateral absence of the vas deferens: the impact of spermatogenesis quality on intracytoplasmic sperm injection outcomes in 108 men. Andrology 2015;3:473-80.

52. Glina S, Fragoso JB, Martins FG, et al. Percutaneous epididymal sperm aspiration (PESA) in men with obstructive azoospermia. Int Braz J Urol 2003;29:141-5; discussion 145-6.

53. Kovac JR, Lehmann KJ, Fischer MA. A single-center study examining the outcomes of percutaneous epididymal sperm aspiration in the treatment of obstructive azoospermia. Urol Ann 2014;6:41-5.

54. Semião-Francisco L, Braga DP, Figueira Rde C, et al. Assisted reproductive technology outcomes in azoospermic men: 10 years of experience with surgical sperm retrieval. Aging Male 2010;13:44-50.

55. Silber SJ, Devroey P, Tournaye H, et al. Fertilizing capacity of epididymal and testicular sperm using intracytoplasmic sperm injection (ICSI). Reprod Fertil Dev 1995;7:281-92; discussion 292-3.

56. Lu S, Cui Y, Li X, et al. Association of cystic fibrosis transmembrane-conductance regulator gene mutation with negative outcome of intracytoplasmic sperm injection pregnancy in cases of congenital bilateral absence of vas deferens. Fertil Steril 2014;101:1255-60.

57. Meniru GI, Gorgy A, Podsiadly BT, et al. Results of percutaneous epididymal sperm aspiration and intracytoplasmic sperm injection in two major groups of patients with obstructive azoospermia. Hum Reprod 1997;12:2443-6.

58. Esteves SC, Lee W, Benjamin DJ, et al. Reproductive potential of men with obstructive azoospermia undergoing percutaneous sperm retrieval and intracytoplasmic sperm injection according to the cause of obstruction. J Urol 
2013;189:232-7.

59. Silber SJ, Balmaceda J, Borrero C, et al. Pregnancy with sperm aspiration from the proximal head of the epididymis: a new treatment for congenital absence of the vas deferens. Fertil Steril 1988;50:525-8.

60. Silber SJ, Van Steirteghem AC, Liu J, et al. High fertilization and pregnancy rate after intracytoplasmic sperm injection with spermatozoa obtained from testicle biopsy. Hum Reprod 1995;10:148-52.

61. Yu J, Chen Z, Ni Y, et al. CFTR mutations in men with congenital bilateral absence of the vas deferens (CBAVD): a systemic review and meta-analysis. Hum Reprod 2012;27:25-35.

62. Naru T, Sulaiman MN, Kidwai A, et al. Intracytoplasmic sperm injection outcome using ejaculated sperm and retrieved sperm in azoospermic men. Urol J 2008;5:106-10.

63. Bernie AM, Ramasamy R, Stember DS, et al. Microsurgical epididymal sperm aspiration: indications, techniques and outcomes. Asian J Androl 2013;15:40-3.

64. Fahmy I, Kamal A, Aboulghar M, et al. Percutaneous aspiration biopsy using an intravenous catheter for testicular sperm retrieval in patients with obstructive azoospermia. Reprod Biomed Online 2004;9:102-5.

65. Hovatta O, Moilanen J, von Smitten K, et al. Testicular needle biopsy, open biopsy, epididymal aspiration

Cite this article as: Persily JB, Vijay V, Najari BB. How do we counsel men with obstructive azoospermia due to $\mathrm{CF}$ mutations?-a review of treatment options and outcomes. Transl Androl Urol 2021;10(3):1467-1478. doi: 10.21037/tau-19681 and intracytoplasmic sperm injection in obstructive azoospermia. Hum Reprod 1995;10:2595-9.

66. Van Peperstraten A, Proctor ML, Johnson NP, et al. Techniques for surgical retrieval of sperm prior to intracytoplasmic sperm injection (ICSI) for azoospermia. Cochrane Database Syst Rev. 2008;2008:CD002807.

67. van der Ven K, Messer L, van der Ven H, et al. Cystic fibrosis mutation screening in healthy men with reduced sperm quality. Hum Reprod 1996;11:513-7.

68. Chen H, Ruan YC, Xu WM, et al. Regulation of male fertility by CFTR and implications in male infertility. Hum Reprod Update 2012;18:703-13.

69. Patrizio P, Ord T, Silber SJ, et al. Cystic fibrosis mutations impair the fertilization rate of epididymal sperm from men with congenital absence of the vas deferens. Hum Reprod 1993;8:1259-63.

70. Meng MV, Black LD, Cha I, et al. Impaired spermatogenesis in men with congenital absence of the vas deferens. Hum Reprod 2001;16:529-33.

71. Ramasamy R, Lipshultz LI. Cystic fibrosis transmembrane regulator mutation and congenital bilateral absence of the vas deferens: a bad combination for successful intracytoplasmic sperm injection outcomes. Fertil Steril 2014;101:1246. 